\title{
Role of Disorder in Mn:GaAs, Cr:GaAs, and Cr:GaN
}

\author{
J. L. Xu and M. van Schilfgaarde \\ Arizona State University, Tempe, AZ, 85284 \\ G. D. Samolyuk \\ Ames Laboratory, Iowa State University, Ames, IA, 50011
}

(Dated: October 22, 2018)

\begin{abstract}
We present calculations of magnetic exchange interactions and critical temperature $T_{c}$ in $\mathrm{Ga}_{1-x} \mathrm{Mn}_{x} \mathrm{As}, \mathrm{Ga}_{1-x} \mathrm{Cr}_{x}$ As and $\mathrm{Ga}_{1-x} \mathrm{Cr}_{x} \mathrm{~N}$. The local spin density approximation is combined with a linear-response technique to map the magnetic energy onto a Heisenberg hamiltonion, but no significant further approximations are made. Special quasi-random structures in large unit cells are used to accurately model the disorder. $T_{c}$ is computed using both a spin-dynamics approach and the cluster variation method developed for the classical Heisenberg model.

We show the following: $(i)$ configurational disorder results in large dispersions in the pairwise exchange interactions; $(i i)$ the disorder strongly reduces $T_{c} ;(i i i)$ clustering in the magnetic atoms, whose tendency is predicted from total-energy considerations, further reduces $T_{c}$. Additionally the exchange interactions $J(R)$ are found to decay exponentially with distance $R^{3}$ on average; and the mean-field approximation is found to be a very poor predictor of $T_{c}$, particularly when $J(R)$ decays rapidly. Finally the effect of spin-orbit coupling on $T_{c}$ is considered. With all these factors taken into account, $T_{c}$ is reasonably predicted by the local spin-density approximation in MnGaAs without the need to invoke compensation by donor impurities.
\end{abstract}


Dilute magnetic semiconductors (DMS), i.e. semiconductors doped with low concentrations of magnetic impurities (usually $\mathrm{Cr}$, Mn, or Co), have attracted much interest because of their potential application to spintronics [1, 2] . $\mathrm{Ga}_{1-x} \mathrm{Mn}_{x}$ As is the most widely studied DMS, and it continues to attract interest because it is one of the few DMS where it is generally agreed that the magnetism is carrier-mediated. (This is important in spintronics because the magnetic state can be manipulated by electrical or optical means.)

In recent years Curie temperatures in $\mathrm{Ga}_{1-x} \mathrm{Mn}_{x} \mathrm{As}$ have risen steadily, reaching $\sim 170 \mathrm{~K}$ for $x \sim 0.08$ when grown in thin films annealed at low temperature [3, 4, 5]. It is generally believed defects (probably Mn interstitials) migrate out of the as-deposited films during the anneal, largely eliminating donor defects that hamper ferromagnetism. Since most practical applications of spintronics require room-temperature operation, a crucial question is then, what is the ultimate limit to $T_{c}$ in the DMS compounds, and in $\mathrm{Ga}_{1-x} \mathrm{Mn}_{x} \mathrm{As}$ in particular?

This question was first addressed by Dietl in his now classic paper [6], where he predicted a wide range of $T_{c}$ in tetrahedrally coordinated alloys. This stimulated a great deal of interest, although there is a growing consensus that most of the claims of that paper were artifacts of the assumptions in his original model. On the other hand, Akai 7] first used the local spin-density approximation (LSDA) to estimate $T_{c}$ within the Coherent Potential Approximation (CPA) in (In,Mn)As; he argued that a double exchange mechanism was a more appropriate description of the magnetism than the $p d$ exchange assumed by Dietl. Since then LSDA calculations of exchange interactions have been performed by a variety of groups [8, 9, 10, 11, 12, 13], usually extracting exchange parameters by calculating total energies of a fixed atomic but multiple-spin configurations, or by a linear-response technique within the CPA.

To date, disorder has almost always been neglected or treated within some mean-field (MF) approximation (MFA), either in the computation of the exchange parameters themselves, or in the subsequent analysis of magnetization $M(T)$ at finite-temperature, or both (though better treatments within $k \cdot p$ theory has been reported [14]). The LSDA+MF predict a rather high $T_{c}$ for $\mathrm{Ga}_{1-x} \mathrm{Mn}_{x} \mathrm{As}$ (typically $350 \sim 400 \mathrm{~K}$ for $x \sim 0.08$ [13]). The large discrepancy with experiment (at least in Mn:GaAs) is usually attributed to the very large numbers of compensating defects in real samples, which reduce $T_{c}[3,44,5]$. The situation remains somewhat uncertain because the number of defects still remaining in the best samples to date is not known. 
This Letter addresses the issue of the ultimate limit to $T_{c}$ in some DMS alloys (focusing on $\mathrm{Mn}: \mathrm{GaAs}$ ) by adopting relatively rigorous approach to the calculation of the magnetic exchange interactions and $T_{c}$. Random alloys are approximated by large (128-250 atom) supercells where special quasirandom structures (SQS) [15] are used for the cation sublattice. Using a linear-response technique within the LSDA and the linear-muffin-tin orbitals method [16, 17], the magnetic energy is mapped onto a Heisenberg form[18]

$$
H=-\sum_{i j} J\left(R_{i j}\right) \hat{e}_{i} \cdot \hat{e}_{j}
$$

where the sum is over all pairs $i j$ of magnetic atoms. To model $M(T)$ and $T_{c}$, Eq.(11) is treated classically and integrated using a spin-dynamics (SD) technique 19]; alternatively $M(T)$ is estimated by the cluster variation method (CVM) 20] adapted 21] to solve Eq.(11). Thus it is evaluated without recourse to empirical parameters or to the MFA. We show that the widely used MFA turns out be a very poor predictor of $M(T)$ in these disorded, dilute alloys, dramatically overestimating $T_{c}$.

With SQS we can rather precisely mimic a fully random configuration, but it is also possible to consider configurations that deviate from random. This can be important because LSDA predicts a strong attractive interaction between magnetic elements [8], which implies a tendency towards clustering. In brief, we show that

- the disorder induces large fluctuations in $J_{i j} \equiv J\left(R_{i j}\right)$ for every connecting vector $R_{i j}$;

- The fluctuations in $J_{i j}$ reduce $T_{c}$ relative to the configurationally averaged $\bar{J}_{i j}=\left\langle J_{i j}\right\rangle$;

- clustering reduces $T_{c}$, while ordering of the magnetic elements increases $T_{c}$.

Fig. 1 shows $J_{i j}$ computed for an ensemble of 108-cation (216-atom) random supercells following the method of Ref.[17], for $\mathrm{Ga}_{1-x} \mathrm{Mn}_{x} \mathrm{As}$ and $\mathrm{Ga}_{1-x} \mathrm{Cr}_{x} \mathrm{~N}$ alloys at $x=4.6 \%$ and $x=8.3 \% .3 \times 3 \times 3 k$-points were used, enabling the calculation of $J$ to very distant neighbors. We chose these two alloys because they are approximately representative of limiting cases. For Cr:GaN, the GaN host has a wide bandgap, and the $\mathrm{Cr} t_{2}$ level falls near midgap. It broadens into an impurity band with 1/3 occupancy, and is believed to be responsible for the ferromagnetic exchange. For Mn:GaAs, most of the weight of the Mn $t_{2}$-derived state falls below the valence band maximum. A second $t_{2}$ impurity band about $0.1 \mathrm{eV}$ above the valence band maximum is mainly responsible for the ferromagnetic exchange coupling 

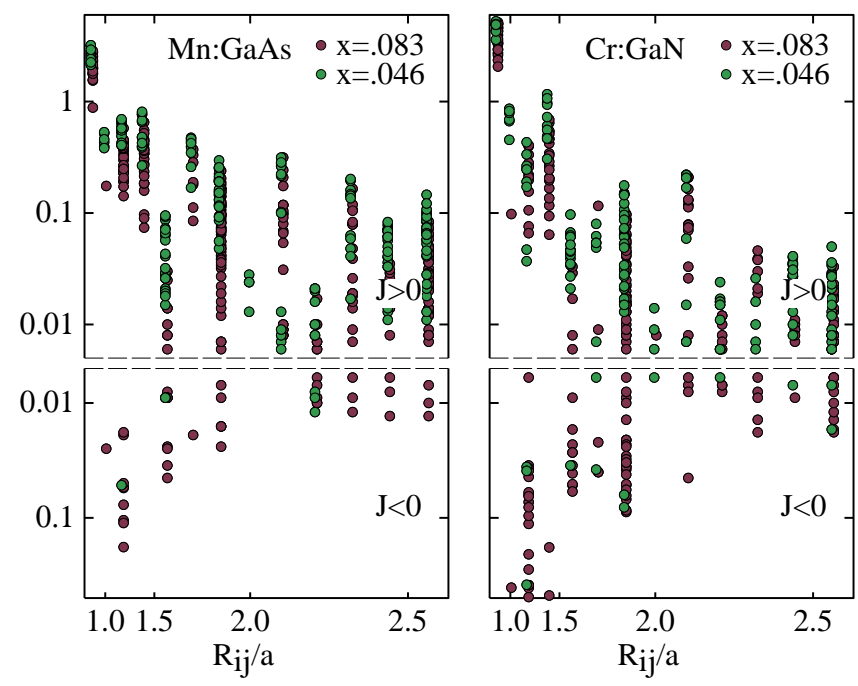

FIG. 1: Pair exchange interactions $J\left(R_{i j}\right)$, in $\mathrm{mRy}$, for $\mathrm{Mn}: \mathrm{GaAs}$ and $\mathrm{Cr}$ :GaN at two different concentrations as a function of $R_{i j}^{3} . R_{i j}$ is measured in units of the lattice constant $a$.

in this case; the strength of $J(R)$ depends critically on the amount of Mn character in this band 22]. Katayama-Yoshida used the $x$-dependence of $J_{0}=\sum_{R} \bar{J}(R)$ (computed within the CPA) to identify the ferromagnetism obtained from LSDA with model theories [13]. Within the CPA, $J_{0} \sim x^{1 / 2}$ for Cr:GaN, which corresponds to a double-exchange model, while Mn:GaAs displays character intermediate between $J_{0} \sim x^{1 / 2}$ and the $p d$ exchange $\left(J_{0} \sim x\right)$ usually assumed by $k \cdot p$ models $[\underline{6}, 23]$.

Comparing Cr:GaN to Mn:GaAs, Cr:GaN shows substantially stronger nearest-neighbor (NN) interactions, owing to its small lattice constant; however $\bar{J}\left(R_{i j}\right)$ decays much more rapidly with $R_{i j}$. This is because the wave function overlap between transition metal $d$ states decays much more rapidly for midgap states than near band-edge states. Evident also is the large dispersion in $J_{i j}$ for fixed $R_{i j}$ (note $J$ is drawn on a log scale): the rootmean square fluctuations $\Delta J_{i j}=\sqrt{\left\langle J_{i j}^{2}-\bar{J}_{i j}^{2}\right\rangle}$ are roughly comparable to $\bar{J}$. However $\Delta J_{i j}$ increases with $x$, and is substantially larger for the wide-gap case (Cr:GaN). Note that there is little evidence in either Cr:GaN or Mn:GaAs for oscillatory RKKY-like behavior, which in the simplest approximation predicts $J(R) \sim \cos \left(2 k_{F} R\right) / R^{3}$. Instead, $\bar{J}(R)$ decays roughly exponentially in $R^{3}$, corresponding to a Fermi surface with imaginary wave number, as would obtain if the coupling were described by tunneling via a disordered impurity band 24 .

We now apply Eq.(11) to compute $M(T)$, focusing on $T_{c}$. Mean-field theory, which estimates the effective field at each site from the average field contributed by other sites, 
predicts $T_{c}$ well above room temperature both in Mn:GaAs and Cr:GaN 13, 25]. In spite of the rather strong differences in the form of $J(R)$ (Fig. 1), mean-field theory predicts that Mn:GaAs and Cr:GaN have roughly similar $T_{c}$ for $x \sim 0.08$ [25]. This is because the NN interaction in the latter case is strongest, but the $J$ decays faster with $R$, leading to a comparable mean-field [26] estimate $\bar{T}_{c}^{M F A}$.

But it should be evident from Fig. 11 that the MFA is of questionable reliability. First, it is well known that for dilute alloys there is a percolation threshold for the onset of ferromagnetism. (The threshold in the present case cannot be readily mapped to known models because $J(R)$ is nonneglible for a rather large number of neighbors.) Moreover, the large fluctuations $\Delta J(R)$ may strongly affect $T_{c}$, especially since $\Delta J(R)$ itself is purely a function of the environment [8], and consequently of the local percolation path.

To obtain a precise estimate for $M(T)$ and $T_{c}$, we adopt a spin-dynamics approach 19]. A 200 atom SQS structure (250 atom for the $4 \%$ alloy) was used to mimic the random alloy. From the TM atoms in the SQS structure, a supercell containing $\sim 2000 \mathrm{Mn}$ or $\mathrm{Cr}$ atoms was constructed to make a simulation cell for prosecuting spin-dynamical simulations. Following the method described in Ref. [19], the Landau-Lifshitz (L-L) equation was integrated numerically at a fixed temperature allowing the system to equilibrate, followed by a simulation for $\sim 2 \times 10^{6}$ atomic units. The L-L equations were integrated with the Bulirsch-Stoer method. As the L-L equation is a first-order equation, global deamons were used for the heat bath [19], to ensure ergodic behavior. The average magnetization $\bar{M}(T)$ was computed as a function of temperature, and $T_{c}$ was estimated from the inflection point in $\bar{M}(T)$. Owing to finite-size effects and the stochastic character of the simulation, $T_{c}$ could be determined to a precision of $\sim 5 \%$.

Also we employed a CVM approach recently adapted to the classical Heisenberg hamiltonion [21]. This relatively simple scheme has been found to be accurate in simple $3 d$ magnets, overestimating $T_{c}$ by $\sim 5 \%$ (similar to the usual CVM for the Ising hamiltonion[27]). We can check the validity both methods in the DMS case by comparing their predictions of $T_{c}$. Fig 2 shows $T_{c}$ determined by both methods for $\mathrm{Ga}_{1-x} \mathrm{Mn}_{x} \mathrm{As}$ and $\mathrm{Ga}_{1-x} \mathrm{Cr}_{x} \mathrm{As}$ : agreement between the two methods is $\sim 10 \%$, which is quite satisfactory considering the complexity of the $J_{i j} \cdot \bar{T}_{c}^{M F A}$ is also shown: evidently the MFA rather badly overestimates $T_{c} \cdot \bar{T}_{c}^{M F A}>T_{c}$ by $\sim 200 \mathrm{~K}$ in the Mn:GaAs alloy, and by a somewhat larger amount in Cr:GaAs. The discrepancy is still more dramatic in Cr:GaN (not shown); we find $T_{c}<50 \mathrm{~K}$ 
for all concentrations studied while $\bar{T}_{c}^{M F A} \sim 600 \mathrm{~K}[25]$. Indeed we have found this generally to be the case when $J(R)$ decays rapidly or when $\Delta J(R) / J(R)$ is not small.

These results stand in stark contrast to the $\sim 15 \%$ discrepancy between $T_{c}^{M F A}$ and $T_{c}$ typically found in simple metals. The reason is easily understood by considering the effective field a mean-field atom sees, $\vec{H}_{i}^{e f f}=\sum_{j} J_{i j} \hat{e}_{j}$. From the exponential decay of $J(R)$, it is evident that $H_{i}$ will be dominated by the nearest neighbors. But for dilute alloys, nearneighbors are not sufficent to form a percolation path. This is immediately evident in the extreme case of a NN pair of magnetic atoms well separated from any other magnetic atoms: the contribution to $T_{c}^{M F A}$ from this pair would be high, even though the pair would actually contribute nothing to ferromagnetism.

In Ref. [10] a small discrepancy between $T_{c}^{M F A}$ and a more sophisticated calculation for $T_{c}$ was reported. In that calculation the CPA was used to construct an average $\bar{J}_{i j}$ and $M(T)$ modeled by constructing a fcc lattice of magnetic atoms, using concentrated-weighted $\bar{J}_{i j}$ for the exchange parameters. It would seem that their conclusions are an artifact of the neglect of configurational disorder (except in the computation of $\bar{J}_{i j}$ ). Better would be to estimate $\bar{J}_{i j}$ within the CPA, and then construct a disordered simulation cell using the $\bar{J}_{i j}$ to estimate $M(T)$. Still this approach neglects fluctuations $\Delta J$, which as we have seen are comparable to $\bar{J}_{i j}$ itself. To assess the effect of fluctuations, we repeated the calculation for $T_{c}$ within the CVM, replacing the environment-specific $J_{i j}$ with the configurationally averaged $\bar{J}_{i j}$. For $\mathrm{Ga}_{1-x} \mathrm{Mn}_{x}$ As at $x=0.08$, the effect of disorder $\left(\bar{J}_{i j} \rightarrow J_{i j}\right)$ was to reduce $T_{c}$ by $50 \mathrm{~K}$. (It is interesting that the MFA predicts the opposite trend, because of an artificial tendency for $M^{M F A}(T)$ to track whichever site $i$ has the largest $\vec{H}_{i}^{e f f}$. Then $T_{c}^{M F A}-\bar{T}_{c}^{M F A}$ is positive 26] and increases with $\Delta J / J$. This explains why a tight-binding + MF analysis 24] predicted that disorder increases $T_{c}$.)

We next consider the effects of nonrandomness. As noted above, real DMS alloys should exhibit some clustering owing to the attractive interaction between magnetic elements[8]. The true situation is complicated by the nonequilibrium growth required to stabilize the alloy in the zincblende structure. Nevertheless the Mn-Mn or $\mathrm{Cr}-\mathrm{Cr}$ binding energy is calculated[8] to be an order of magnitude larger than the growth temperature $(\sim 250 \mathrm{~K})$, and some pairing or other clustering should be expected, particularly since films must be annealed to obtain $\operatorname{good} T_{c}$. There is some experimental evidence for a tendency to cluster [28].

The effect of clustering on $T_{c}$ in $\mathrm{Ga}_{0.92} \mathrm{Mn}_{0.08} \mathrm{As}$ was studied by a simple model. To 


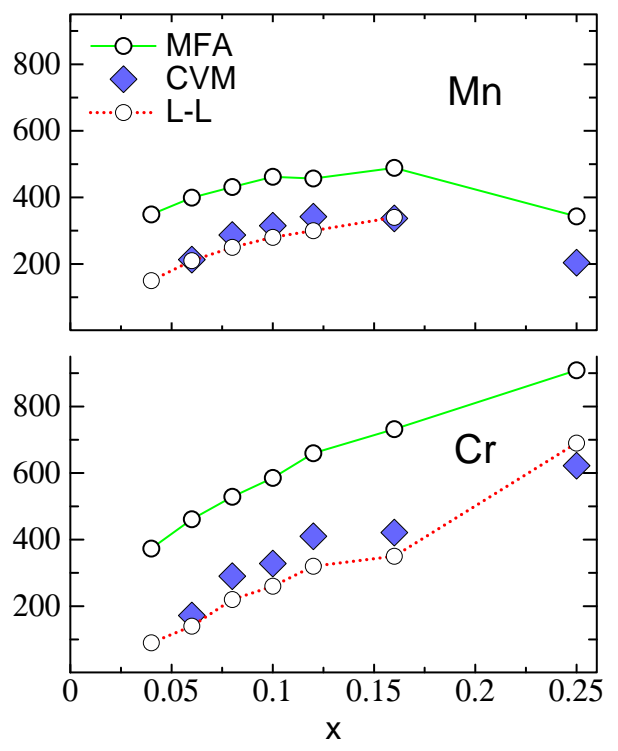

FIG. 2: Dependence of $T_{c}(\mathrm{~K})$ on $x$ in $\mathrm{Ga}_{1-x} \mathrm{Mn}_{x}$ As and $\mathrm{Ga}_{1-x} \mathrm{Cr}_{x}$ As. Solid lines: $T_{c}$ computed from the $\mathrm{MF} \bar{T}_{c}^{M F A}[26]$. Dotted line: $T_{c}$ extracted from spin-dynamics simulations of Eq. [1, Diamonds: $T_{c}$ computed from the Heisenberg Cluster Variation Method.

characterize the configurational disorder we adopt the standard Ising formalism, and assign $\sigma= \pm 1$ to each cation site ( +1 for $\mathrm{Mn}$ and -1 for $\mathrm{Ga}$ ). The random (SQS) configuration was constructed by searching for configurations which best approximate the ideal random configuration for pair correlation functions $P_{R_{i j}}=\left\langle\sigma_{i} \sigma_{j}\right\rangle$ (and some higher-order correlation functions) up to some fixed distance. For a random configuration, $P_{R}=(2 x-1)^{2}$ independent of $R$. To parameterize the clustering in a simple manner, we adopted the NN pair correlation function $P_{1}$ as a measure of clustering. Starting from an initial SQS configuration, a simulated annealing cycle was performed by generating a set of site configurations with increasing $P_{1}$, corresponding to longer annealing times (For simplicity, $P_{n}(n>1)$ was optimized to be $(2 x-1)^{2}$ for each configuration.) $J_{i j}$ and $T_{c}$ were computed by the CVM and MFA [26] as a function of $P_{1}$; see Fig. 3. $T_{c}$ is rather strongly reduced with increasing $P_{1}$. This is perhaps not surprising since increased clustering implies more distant average separation between atoms, which is deleterious to links in the percolation path. Even within the MFA $T_{c}$ changes slightly, albeit for a different reason. In that case, there is an increase in NN pairs, which would increase $T_{c}$, but at the same time there is some increase in the likelihood of three- and higher body neighbors. The presence of a third neighbor has the effect of reducing the pairwise $J_{i j}$ [8], and is the origin of the factor-of-three variations in 
the NN $J$ in Fig. 1]

We also considered the ordered limit, by putting $1 \mathrm{Mn}$ in a 24-atom unit cell, corresponding to $x=0.083$. In this case $P_{1}$ decreases to $2 / 3$, and $T_{c}$ increases to $350 \mathrm{~K}$ (see Fig. (3). Thus we conclude that ordering increases $T_{c}$, while clustering decreases $T_{c}$. Perhaps not suprisingly, the MFA $T_{c}$ approaches the CVM result in the ordered case, since percolation is less critical.

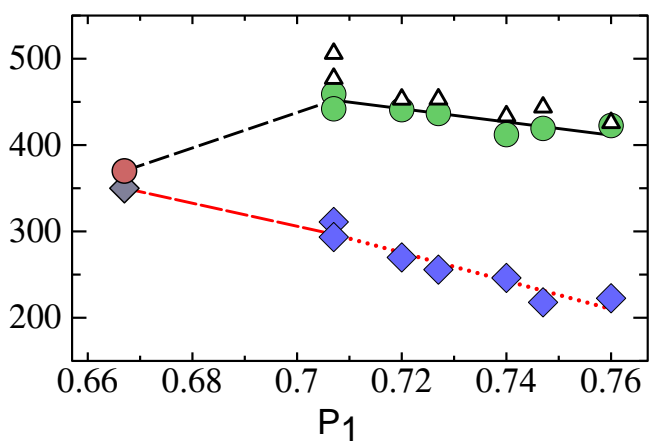

FIG. 3: Dependence of $T_{c}(\mathrm{~K})$ on the pair correlation function $P_{1}$ in $\mathrm{Ga}_{0.92} \mathrm{Mn}_{0.08}$ As. The random (SQS) configuration corresponds to $P_{1}=0.7056$. (Two SQS structures were calculated.) Diamonds show $T_{c}$ computed with CVM; circles show $\bar{T}_{c}^{M F A}$, and triangles show $T_{c}^{M F A}$. The point at $P_{1}=2 / 3$ corresponds to the ordered compound.

To conclude, we have shown that ferromagnetism is very sensitive to configurational disorder in DMS alloys, and that with proper treatment of disorder $T_{c}$ is reasonably predicted by the LSDA for $\mathrm{Ga}_{1-x} \mathrm{Mn}_{x}$ As, without needing to invoke compensating defects. We briefly consider two important sources of error from elements missing in the theory. First, spin-orbit coupling strongly reduces $T_{c}$ in $k \cdot p$ models. We estimated its effect by computing the change in $\bar{T}_{c}^{M F A}$ when the $L \cdot S$ coupling is added to the LSDA hamiltonion. For $\mathrm{Ga}_{0.92} \mathrm{Mn}_{0.08} \mathrm{As}$, $\bar{T}_{c}^{M F A}$ was reduced by $\sim 10 \%$. Finally, the LSDA itself will overestimate $T_{c}$ somewhat [22]. In a future work we will present a reliable parameter-free theory that corrects the principal errors in LSDA - most importantly the Mn $d$ character at $E_{F}$-and quantify the extent to which the LSDA overestimates $T_{c}$. Finally, we conclude that the present calculations represent a rather strict upper bound to $T_{c}$, and that for random or clustered $\mathrm{Ga}_{1-x} \mathrm{Mn}_{x} \mathrm{As}$ alloys, $T_{c}>250 \mathrm{~K}$ is unlikely.

This work was supported by the Office of Naval Research. 
[1] H. Ohno, A. Shen, F. Matsukura, A. Oiwa, A. Endo, S. Katsumoto, and Y. Iye, Applied Physics Letters 69, 363 (1996).

[2] Y. Ohno, D. K. Young, B. Beschoten, F. Matsukura, H. Ohno, and D. D. Awschalom, Nature 402, 790 (1999).

[3] D. Chiba, K. Takamura, F. Matsukura, and H. Ohno, Appl. Phys. Lett. 82, 3020 (2003).

[4] K. C. Ku, S. J. Potashnik, R. F. Wang, S. H. Chun, P. Schiffer, N. Samarth, M. J. Seong, A. Mascarenhas, E. Johnston-Halperin, R. C. Myers, et al., Appl. Phys. Lett. 82, 2302 (2003).

[5] K. W. Edmonds, P. Bogusawski, K. Y. Wang, R. P. Campion, S. N. Novikov, N. R. S. Farley, B. L. Gallagher, C. T. Foxon, M. Sawicki, T. Dietl, et al., Phys. Rev. Lett. 92, 037201 (2004).

[6] T. Dietl, H. Ohno, F. Matsukura, J. Cibert, and D. Ferrand, Science 287, 1019 (2002).

[7] H. Akai, Phys. Rev. Lett. 81, 3002 (1998).

[8] M. van Schilfgaarde and O. Mryasov, Phys. Rev. B 63, 233205 (2001).

[9] L. M. Sandratskii and P. Bruno, Phys. Rev. B 66, 134435 (2002).

[10] G. Bouzerar, J. Kudrnovský, L. Bergqvist, and P. Bruno, Phys. Rev. B 68, 081203(R) (2003).

[11] J. Kudrnovský, I. Turek, V. Drchal, F. Maca, P. Weinberger, , and P. Bruno, Phys. Rev. B 69, 115208 (2004).

[12] S. C. Erwin and C. S. Hellberg, Phys. Rev. B 68, 245206 (2003).

[13] M. Sato, H. Katayama-Yoshida, and P. Dederics, Europhys. Lett. 61, 403 (2003).

[14] J. Schliemann, J. König, H.-H. Lin, and A. H. MacDonald, Appl. Phys. Lett. 78, 1550 (2001).

[15] A. Zunger, S.-H. Wei, L. G. Ferreira, and J. E. Bernard, Phys. Rev. Lett. 65, 353 (1990).

[16] A. I. Liechtenstein, M. I. Katsnelson, V. P. Antropov, and V. A. Gubanov, J. Magn. Magn. Mater. 67, 65 (1987).

[17] M. van Schilfgaarde and V. P. Antropov, J. Appl. Phys. 85, 4827 (1999).

[18] Because the TM magnetic moments are large, the local-moment picture and long-wave approximation is expected to be rather accurate; see [? ].

[19] V. P. Antropov, M. I. Katsnelson, B. N. Harmon, M. van Schilfgaarde, and D. Kusnezov, Phys. Rev. B 54, 1019 (1996).

[20] R. Kikuchi, Phys. Rev. 81, 988 (1951).

[21] In contrast to the Ising model[27], the classical Heisenberg model has continuous rotational 
degrees of freedom which leads to a more complicated form for the partition function: $Z_{i j}=\sum_{l}(2 l+1) i_{l}\left(\psi_{i}^{i j} / T\right) i_{l}\left(J_{i j} / T\right) i_{l}\left(\psi_{j}^{i j} / T\right)$, where $i_{l}(z)=\sqrt{(\pi / 2 z)} I_{l+1 / 2}(z)$ and $I_{n}(z)$ is the modified Bessel function of the first kind. Cluster methods can include strong correlation among atoms, which the MFA neglects. The CVM accurately describes interactions within the clusters (pair clusters in our case). The interactions of the cluster with the environment is described by renormalizing fields $\psi^{i j}$.

[22] P. Mahadevan and A. Zunger, Phys. Rev. B 69, 115211 (2004).

[23] T. Jungwirth, W. A. Atkinson, B. H. Lee, and A. H. MacDonald, Phys. Rev. B 59, 981 (1999).

[24] M. Berciu and R. N. Bhatt, Phys. Rev. Lett. 87, 107203 (2001).

[25] S. Wu, H. Liu, L. Gu, R. Singh, L. Budd, M. van Schilfgaarde, M. McCartney, D. J. Smith, and N. Newman, Appl. Phys. Lett. 82, 3047 (2003).

[26] Mean-field theory for the Heisenberg model approximates the coupling to neighboring sites $j$ by an effective field $\vec{H}_{i}^{e f f}=\sum_{j} J_{i j} \hat{e}_{j}$ at site $i$, and entails the solution of a set of coupled nonlinear equations in $H_{i}$ and $\hat{e}_{i}$. Alternatively, we can replace $J_{i j}$ with its average $\bar{J}_{i j}$ and then calculate $M(T)$ for the $\bar{J}_{i j}$. We call $T_{c}$ computed by the two approaches respectively, $T_{c}^{M F A}$ and $\bar{T}_{c}^{M F A}$. The latter can be easily evaluated as $\bar{T}_{c}^{M F A}=2 / 3\left\langle\sum_{i j} J_{i j}\right\rangle$.

[27] V. G. Vaks and G. D. Samolyuk, Sov. Phys. : JETP 88, 89 (1999).

[28] J. M. Sullivan, G. I. Boishin, L. J. Whitman, A. T. Hanbicki, B. T. Jonker, and S. C. Erwin, Phys. Rev. B 68, 235324 (2003). 